\title{
POLIOMYELITIS IN ENGLAND AND WALES, 1947-1950
}

\author{
BY \\ W. J. MARTIN \\ Medical Research Council's Statistical Research Unit, \\ London School of Hygiene and Tropical Medicine
}

Poliomyelitis has been endemic in England and Wales since its notification was made compulsory in 1912, but prior to 1947 its incidence was, with the exception of three years, of the nature of a sporadic disease with a seasonal rise rather than that of an epidemic disease. This was in contrast to the experience of the United States and some continental countries, where large epidemics had occurred periodically. On the other hand, the vagaries of the disease in England and Wales between the two wars presented some interesting and puzzling features. The fatality rate, i.e. the proportion of deaths to notified cases, varied greatly from year to year; for example, the notifications in 1926 were three times the 1925 level but the fatality rate only half. On the whole, the yearly deaths from poliomyelitis varied only slightly in number, and the concomitant large variations in the notifications would (if they can be accepted as accurate) suggest that the severity of the disease was changing from year to year. Similarly, the incidence of notifications increased more than the mortality during the seasonal period of greater prevalence. This was possibly due to an increased recognition of milder cases at the times when the disease was known to be most likely to occur. The Registrar-General, discussing this possibility, wrote:

There is evidence that milder cases of the disease are often overlooked, its characteristic after-effects being met with in persons in whom the early stages of the disease have never been recognized.

The only large outbreaks of the disease in England and Wales before the epidemics of 1947-50 occurred in 1926, 1938, and 1940, when 1,159,1,489, and 1,079 cases were notified. From these occurrences the outbreak of 1947 differed not only in magnitude- 708 cases were notified in the week of maximum prevalence -but also in the geographical and age distribution of the patients. This outbreak, in fact, appears to mark a distinct change in the incidence of poliomyelitis in England and Wales, for though a steep decline was recorded in 1948, the incidence remained considerably above the previous endemic level and the year's notifications were larger than those of any year prior to 1947. Any hopes that poliomyelitis. would follow a similar trend to that exhibited by cerebrospinal fever after the epidemic of 1941 (when the succeeding outbreaks gradually diminished in size until an endemic level slightly above the original endemic level was reached) were: disappointed when large outbreaks of poliomyelitis appeared in 1949 and 1950. 
Characteristics of the recent outbreaks have been an apparent change in infectivity and concentration of the disease. It has always been believed that the occurrence of multiple cases of poliomyelitis in a family or even in a household was a very rare event. During the last epidemics two cases in a household were not infrequently reported, and in 1949 three cases of the disease occurred in each of three households in the Isle of Wight (Bradley, 1950). In earlier outbreaks, centres of high incidence generally occurred and there was not much evidence of a spread of infection. Thus in 1926 the neighbouring counties of Leicester, Nottingham, and Warwick contributed 21 per cent. of the total notifications of the country, the population of this area being only 6.7 per cent. of that of the whole country. In 1938, the notifications in London and Essex were 21 per cent. of the total, the population being 14.5 per cent. of the total; in 1940, 34 per cent. of the total cases were notified in Lancashire and Yorkshire West Riding where the population was $13 \cdot 2$ per cent. of that of the whole country. On the other hand, the concentration of the bulk of the cases in a limited area practically disappeared in the recent epidemics, although in 1950 the counties of Warwick, Gloucester, and Stafford had 19 per cent. of the total notifications of the country. The regional distribution for the last 4 years is shown in Table I. The chief features of Table I are the light incidence in 1949 in Wales (particularly North Wales) and in the northern counties, and the high attack rate in 1950 in the west midland counties.

TABLE I

Regional Distributions

\begin{tabular}{|c|c|c|c|c|c|c|c|c|c|}
\hline \multirow{2}{*}{\multicolumn{2}{|c|}{ Region }} & \multicolumn{4}{|c|}{ Notifications per Million } & \multicolumn{4}{|c|}{ Rate as Percentage of Total } \\
\hline & & 1947 & 1948 & 1949 & 1950 & 1947 & 1948 & 1949 & 1950 \\
\hline 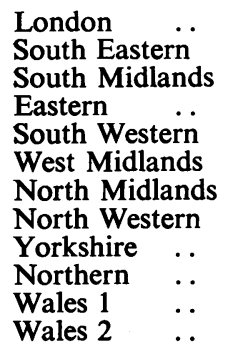 & $\begin{array}{l}. . \\
\ldots \\
\ldots \\
\ldots \\
\ldots \\
. . \\
\ldots \\
. .\end{array}$ & $\begin{array}{l}326 \\
249 \\
218 \\
163 \\
162 \\
208 \\
205 \\
209 \\
230 \\
272 \\
153 \\
173\end{array}$ & $\begin{array}{l}61 \\
51 \\
73 \\
49 \\
73 \\
52 \\
49 \\
38 \\
40 \\
29 \\
57 \\
35\end{array}$ & $\begin{array}{r}264 \\
173 \\
172 \\
191 \\
211 \\
111 \\
163 \\
110 \\
188 \\
85 \\
96 \\
35\end{array}$ & $\begin{array}{l}172 \\
223 \\
152 \\
165 \\
295 \\
386 \\
206 \\
129 \\
168 \\
196 \\
141 \\
238\end{array}$ & $\begin{array}{r}148 \\
113 \\
99 \\
74 \\
73 \\
94 \\
93 \\
95 \\
104 \\
123 \\
69 \\
78\end{array}$ & $\begin{array}{r}120 \\
100 \\
143 \\
96 \\
143 \\
102 \\
96 \\
75 \\
78 \\
57 \\
112 \\
69\end{array}$ & $\begin{array}{r}168 \\
110 \\
110 \\
122 \\
134 \\
71 \\
104 \\
70 \\
120 \\
54 \\
61 \\
22\end{array}$ & $\begin{array}{r}82 \\
106 \\
72 \\
79 \\
140 \\
184 \\
98 \\
61 \\
80 \\
93 \\
67 \\
113\end{array}$ \\
\hline $\begin{array}{c}\text { Total England and } \\
\text { Wales }\end{array}$ & & 221 & 51 & 157 & 210 & 100 & 100 & 100 & 100 \\
\hline
\end{tabular}

\section{MORTALITY}

The age distribution of the deaths from poliomyelitis in 1947 showed a distinct shift compared with earlier outbreaks. The youngest ages formerly supplied the bulk of the deaths, but in 1947 the disease took a much increased toll of the early middle ages. The percentage of deaths under the age of 20 and between the ages of 20 and 45 were 80 and 17 in 1926, 74 and 22 in 1938, and 55 and 41 in 1947. 
This change in the age distribution of the deaths was accompanied by an increase in the ratio of male to female deaths. Male deaths expressed as a percentage of female deaths were 118 in 1926,112 in 1938 , and 145 in 1947 . The fatality rate fell in the 1947 epidemic, for the notifications in this year were 6.6 and 5.2 times the totals of 1926 and 1938 , but the deaths were only 2.9 and 2.7 times as many. The fatality rate rose with age during the more recent outbreaks, the increase being larger for males than for females. The rates are shown in Table II.

TABLE II

Deaths as Percentage of Corrected Notifications, England and Wales

\begin{tabular}{|c|c|c|c|c|c|c|}
\hline \multirow{2}{*}{ Ages } & \multicolumn{2}{|c|}{1947} & \multicolumn{2}{|c|}{1948} & \multicolumn{2}{|c|}{1949} \\
\hline & Males & Females & Males & Females & Males & Females \\
\hline $\begin{array}{c}0- \\
5- \\
10- \\
15- \\
25+\end{array}$ & $\begin{array}{r}5 \cdot 8 \\
5 \cdot 2 \\
8 \cdot 0 \\
13 \cdot 7 \\
21 \cdot 8\end{array}$ & $\begin{array}{r}5 \cdot 0 \\
5 \cdot 2 \\
6 \cdot 5 \\
10 \cdot 1 \\
16 \cdot 1\end{array}$ & $\begin{array}{r}2 \cdot 7 \\
10 \cdot 4 \\
8 \cdot 6 \\
25 \cdot 9 \\
32 \cdot 3\end{array}$ & $\begin{array}{r}5 \cdot 5 \\
6 \cdot 2 \\
6 \cdot 7 \\
20 \cdot 1 \\
23 \cdot 6\end{array}$ & $\begin{array}{r}5 \cdot 8 \\
7 \cdot 9 \\
7 \cdot 7 \\
14 \cdot 1 \\
30 \cdot 4\end{array}$ & $\begin{array}{r}6 \cdot 2 \\
6 \cdot 2 \\
7 \cdot 9 \\
18 \cdot 2 \\
23 \cdot 7\end{array}$ \\
\hline
\end{tabular}

NotifiCATIONS

The epidemic curves for 1947, 1949, and 1950 varied in shape. In 1947 the notifications rose rapidly from the 23 rd week to a maximum of 691 in the 33rd week, declined for two weeks and then, after a further increase to 708 , steadily declined to the end of the year. In 1949 the rise to a peak was much slower and more irregular and the maximum incidence of 563 was not reached until the 41 st week, from which time there was a continuous decline. In 1950 the outbreak began earlier, but the peak incidence occurred in much the same period as in 1947, the maximum being attained in the 34th week with 589 cases; the subsequent decline was interrupted by occasional small rises. The three epidemics show asymmetrical distributions. Their course is shown in the Figure.

\section{Regional Distribution}

The notification rates per million for 1947-50 are shown for each county, and for county boroughs, urban districts, and rural districts of each county, in Table III. For England and Wales as a whole there is, it will be seen, very little difference in incidence between the three density areas. Within the counties there are occasional large differences between the types of area, due to intensive outbreaks in particular areas. Thus, in 1947, the rural districts of Surrey had an incidence rate slightly over twice that of the county as a whole, a feature due mainly to an outbreak in Hambledon R.D. with a rate of 1,148 per million. Similarly, the high rate in the urban districts of Bedfordshire in 1947 was mainly due to the unfavourable experience of the town of Luton. Other small areas with a high attack rate in that year included the rural districts of Aysgarth and Bedale in the North Riding of Yorkshire, and Shildon U.D. in the county of Durham. The chief feature of the distribution of the notifications in 1949 was the low incidence in the northern 


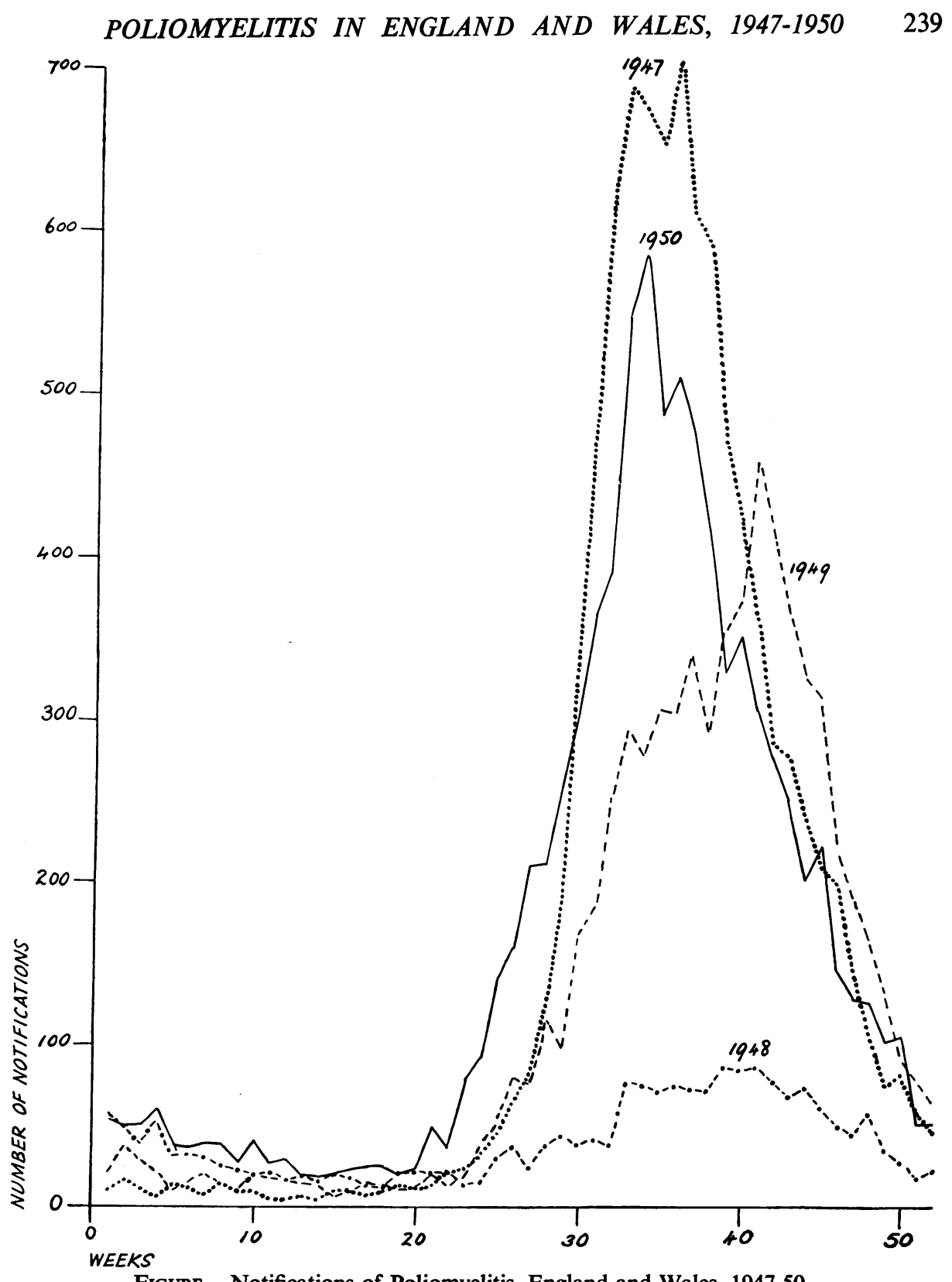

FIGURE.-Notifications of Poliomyelitis, England and Wales, 1947-50.

counties and in Wales, particularly North Wales. The large attack rate in Cornwall was due to an outbreak extending over the districts of Camborne-Redruth U.D., Truro M.B., and Truro R.D.; with a combined rate of 780 per million. Bristol had the largest epidemic among the county boroughs, and the experience 
TABLE

ACute Poliomyelitis in Notifications

\begin{tabular}{|c|c|c|c|c|c|c|c|c|c|c|}
\hline \multirow{2}{*}{\multicolumn{7}{|c|}{ Counties }} & \multicolumn{4}{|c|}{1947} \\
\hline & & & & & & & \multirow{2}{*}{$\frac{\text { County }}{326}$} & \multirow[t]{2}{*}{ C.B.s } & \multirow[t]{2}{*}{ U.D.s } & \multirow[t]{2}{*}{ R.D.s } \\
\hline LONDON & . & . & .. & . & . & .. & & & & \\
\hline & $\begin{array}{l}\text { Surrey } \\
\text { Kent .. } \\
\text { Sussex } \\
\text { Hants } \\
\text { Berks. .. }\end{array}$ & $\begin{array}{l}\ldots \\
\ldots \\
\cdots \\
\ldots\end{array}$ & $\begin{array}{l}. \\
\cdots \\
\cdots \\
\cdots\end{array}$ & $\begin{array}{l}. \\
\ldots \\
. \\
\ldots\end{array}$ & $\begin{array}{l}. \\
\ldots \\
\cdots \\
\ldots\end{array}$ & $\begin{array}{l}. . \\
. \\
. \\
. \\
.\end{array}$ & $\begin{array}{l}292 \\
241 \\
248 \\
226 \\
174\end{array}$ & $\begin{array}{r}318 \\
123 \\
252 \\
184 \\
88\end{array}$ & $\begin{array}{l}248 \\
243 \\
214 \\
238 \\
208\end{array}$ & $\begin{array}{l}617 \\
242 \\
285 \\
293 \\
212\end{array}$ \\
\hline \multirow[t]{2}{*}{ SOUTH EASTERN } & . & .. & .. & .. & .. & .. & 249 & 217 & 240 & 304 \\
\hline & $\begin{array}{l}\text { Middlesex } \\
\text { Hertford } \\
\text { Bucks. } \\
\text { Oxford } \\
\text { Northants } \\
\text { Hunts. } \\
\text { Bedford } \\
\text { Cambridge }\end{array}$ & $\begin{array}{l}. \\
\ldots \\
\ldots \\
. \\
. \\
\ldots \\
\ldots \\
\ldots\end{array}$ & $\begin{array}{l}. . \\
\cdots \\
\ddot{.} \\
\cdots \\
\cdots \\
\cdots \\
.\end{array}$ & $\begin{array}{l}\ldots \\
\ldots \\
\ldots \\
\ldots \\
\ldots \\
\ldots \\
\ldots \\
.\end{array}$ & $\begin{array}{l}\ldots \\
\ldots \\
\ldots \\
\ldots \\
\ldots \\
\cdots \\
\cdots \\
\cdots\end{array}$ & $\begin{array}{l}. . \\
. \\
. \\
. \\
. \\
. \\
. \\
.\end{array}$ & $\begin{array}{r}237 \\
236 \\
130 \\
212 \\
133 \\
149 \\
386 \\
93\end{array}$ & $\begin{array}{l}212 \\
224\end{array}$ & $\begin{array}{r}237 \\
233 \\
136 \\
242 \\
56 \\
214 \\
458 \\
103\end{array}$ & $\begin{array}{r}245 \\
125 \\
200 \\
171 \\
92 \\
217 \\
81\end{array}$ \\
\hline \multirow[t]{2}{*}{ South Midlands } & . & .. & .. & .. & . & .. & 218 & 218 & 230 & 168 \\
\hline & $\begin{array}{l}\text { Essex } \\
\text { Suffolk } \\
\text { Norfolk }\end{array}$ & $\begin{array}{l}. \\
. \\
.\end{array}$ & $\begin{array}{l}. \\
\cdots \\
.\end{array}$ & $\begin{array}{l}. \\
\cdots \\
.\end{array}$ & $\begin{array}{l}. \\
\cdots \\
.\end{array}$ & $\begin{array}{l}. . \\
. .\end{array}$ & $\begin{array}{l}156 \\
170 \\
182\end{array}$ & $\begin{array}{r}102 \\
60 \\
263\end{array}$ & $\begin{array}{l}166 \\
218 \\
203\end{array}$ & $\begin{array}{l}207 \\
195 \\
128\end{array}$ \\
\hline \multirow[t]{2}{*}{ EASTERN } & .. & . & . & .. & . & .. & 163 & 134 & 172 & 171 \\
\hline & $\begin{array}{l}\text { Wilts. } \\
\text { Dorset } \\
\text { Devon } \\
\text { Cornwall } \\
\text { Somerset }\end{array}$ & $\begin{array}{l}\ldots \\
\ldots \\
\cdots \\
. \\
.\end{array}$ & $\begin{array}{l}\cdots \\
\cdots \\
\cdots \\
\cdots\end{array}$ & $\begin{array}{l}\cdots \\
\cdots \\
\cdots \\
\cdots\end{array}$ & $\begin{array}{l}\ldots \\
\cdots \\
\cdots \\
\ldots\end{array}$ & $\begin{array}{l}. . \\
. \\
. \\
.\end{array}$ & $\begin{array}{l}198 \\
289 \\
112 \\
138 \\
161\end{array}$ & $\begin{array}{r}86 \\
235\end{array}$ & $\begin{array}{l}129 \\
256 \\
124 \\
168 \\
110\end{array}$ & $\begin{array}{l}267 \\
347 \\
128 \\
102 \\
186\end{array}$ \\
\hline \multirow[t]{2}{*}{ SOUTH WeSTERN } & . & . & .. & .. & .. & .. & 162 & 120 & 152 & 190 \\
\hline & $\begin{array}{l}\text { Gloucester } \\
\text { Hereford } \\
\text { Salop .. } \\
\text { Staffs. . } \\
\text { Worcester } \\
\text { Warwick }\end{array}$ & $\begin{array}{l}\ldots \\
\cdots \\
\cdots \\
\cdots \\
\cdots\end{array}$ & $\begin{array}{l}. \\
\ldots \\
\cdots \\
\cdots \\
\cdots\end{array}$ & $\begin{array}{l}\because \\
\cdots \\
\cdots \\
\cdots \\
.\end{array}$ & $\begin{array}{l}\because \\
\because \\
\cdots \\
\cdots \\
.\end{array}$ & $\begin{array}{l}. . \\
. \\
. \\
. \\
.\end{array}$ & $\begin{array}{l}216 \\
398 \\
140 \\
151 \\
160 \\
265\end{array}$ & $\begin{array}{r}156 \\
90 \\
257\end{array}$ & $\begin{array}{l}164 \\
302 \\
142 \\
136 \\
140 \\
311\end{array}$ & $\begin{array}{l}235 \\
465 \\
139 \\
177 \\
284 \\
244\end{array}$ \\
\hline \multirow[t]{2}{*}{ West Midlands } & .. & .. & .. & .. & .. & .. & 208 & 215 & 180 & 234 \\
\hline & $\begin{array}{l}\text { Leicester } \\
\text { Rutland } \\
\text { Lincs. } \\
\text { Notts. .. } \\
\text { Derby .. }\end{array}$ & $\begin{array}{l}\cdots \\
\cdots \\
\cdots \\
\cdots\end{array}$ & $\begin{array}{l}\cdots \\
\cdots \\
\cdots \\
\cdots\end{array}$ & $\begin{array}{l}\cdots \\
\ldots \\
\cdots \\
\ldots\end{array}$ & $\begin{array}{l}\cdots \\
\cdots \\
\cdots \\
\cdots\end{array}$ & $\begin{array}{l}. \\
\cdots \\
\cdots \\
. .\end{array}$ & $\begin{array}{l}232 \\
158 \\
336 \\
120 \\
162\end{array}$ & $\begin{array}{r}386 \\
348 \\
65 \\
158\end{array}$ & $\begin{array}{l}103 \\
279 \\
247 \\
140 \\
177\end{array}$ & $\begin{array}{l}103 \\
130 \\
402 \\
182 \\
146\end{array}$ \\
\hline NORTH MIDLANDS & .. & . & .. & .. & .. & .. & 205 & 234 & 169 & 219 \\
\hline
\end{tabular}

of this city adversely affected the rate for Gloucestershire. In 1950 there was a definite peak of cases in the west midlands. Within this area the highest rates 
III

ENGLAND AND WALES, $1947-1950$

per $1,000,000$

\begin{tabular}{|c|c|c|c|c|c|c|c|c|c|c|c|}
\hline \multicolumn{4}{|c|}{1948} & \multicolumn{4}{|c|}{1949} & \multicolumn{4}{|c|}{1950} \\
\hline County & C.B.s & U.D.s & R.D.s & County & C.B.s & U.D.s & R.D.s & County & C.B.s & U.D.s & R.D.s \\
\hline 61 & & & & 264 & & & & 172 & & & \\
\hline $\begin{array}{l}55 \\
58 \\
36 \\
47 \\
56\end{array}$ & $\begin{array}{l}53 \\
79 \\
11 \\
49 \\
44\end{array}$ & $\begin{array}{l}54 \\
58 \\
32 \\
35 \\
75\end{array}$ & $\begin{array}{l}70 \\
55 \\
64 \\
61 \\
54\end{array}$ & $\begin{array}{l}129 \\
208 \\
136 \\
184 \\
259\end{array}$ & $\begin{array}{r}68 \\
113 \\
155 \\
170 \\
329\end{array}$ & $\begin{array}{l}138 \\
198 \\
107 \\
189 \\
251\end{array}$ & $\begin{array}{l}163 \\
254 \\
151 \\
206 \\
221\end{array}$ & $\begin{array}{l}152 \\
204 \\
134 \\
425 \\
136\end{array}$ & $\begin{array}{r}156 \\
302 \\
83 \\
289 \\
69\end{array}$ & $\begin{array}{l}137 \\
213 \\
170 \\
638 \\
115\end{array}$ & $\begin{array}{l}272 \\
159 \\
141 \\
362 \\
185\end{array}$ \\
\hline 51 & 41 & 51 & 60 & 173 & 159 & 167 & 202 & 223 & 195 & 235 & 218 \\
\hline $\begin{array}{r}69 \\
63 \\
97 \\
60 \\
94 \\
0 \\
97 \\
63\end{array}$ & $\begin{array}{l}86 \\
67\end{array}$ & $\begin{array}{r}69 \\
54 \\
121 \\
21 \\
126 \\
0 \\
124 \\
94\end{array}$ & $\begin{array}{r}87 \\
72 \\
53 \\
71 \\
0 \\
33 \\
26\end{array}$ & $\begin{array}{r}179 \\
215 \\
136 \\
153 \\
153 \\
209 \\
189 \\
91\end{array}$ & $\begin{array}{l}186 \\
105\end{array}$ & $\begin{array}{r}179 \\
189 \\
129 \\
125 \\
188 \\
34 \\
123 \\
138\end{array}$ & $\begin{array}{r}282 \\
142 \\
135 \\
155 \\
346 \\
332 \\
34\end{array}$ & $\begin{array}{r}140 \\
151 \\
172 \\
124 \\
284 \\
90 \\
146 \\
68\end{array}$ & $\begin{array}{r}93 \\
153\end{array}$ & $\begin{array}{r}140 \\
147 \\
161 \\
209 \\
350 \\
68 \\
161 \\
62\end{array}$ & $\begin{array}{r}162 \\
184 \\
119 \\
294 \\
106 \\
114 \\
76\end{array}$ \\
\hline 73 & 76 & 76 & 58 & 172 & 146 & 171 & 185 & 152 & 123 & 151 & 161 \\
\hline $\begin{array}{l}43 \\
28 \\
87\end{array}$ & $\begin{array}{r}50 \\
0 \\
137\end{array}$ & $\begin{array}{l}41 \\
44 \\
71\end{array}$ & $\begin{array}{l}41 \\
32 \\
61\end{array}$ & $\begin{array}{l}207 \\
142 \\
171\end{array}$ & $\begin{array}{r}121 \\
58 \\
159\end{array}$ & $\begin{array}{l}218 \\
153 \\
154\end{array}$ & $\begin{array}{l}310 \\
179 \\
183\end{array}$ & $\begin{array}{r}153 . \\
71 \\
286\end{array}$ & $\begin{array}{r}123 \\
58 \\
201\end{array}$ & $\begin{array}{r}160 \\
66 \\
308\end{array}$ & $\begin{array}{r}166 \\
82 \\
331\end{array}$ \\
\hline 49 & 63 & 43 & 47 & 191 & 121 & 209 & 222 & 165 & 132 & 159 & 211 \\
\hline $\begin{array}{r}121 \\
77 \\
47 \\
57 \\
85 \\
\end{array}$ & $\begin{array}{l}49 \\
90 \\
\end{array}$ & $\begin{array}{r}138 \\
82 \\
38 \\
49 \\
90 \\
\end{array}$ & $\begin{array}{r}103 \\
69 \\
54 \\
67 \\
79\end{array}$ & $\begin{array}{l}205 \\
255 \\
149 \\
340 \\
201\end{array}$ & $\begin{array}{l}106 \\
272\end{array}$ & $\begin{array}{l}201 \\
254 \\
149 \\
454 \\
165\end{array}$ & $\begin{array}{l}207 \\
256 \\
200 \\
202 \\
212\end{array}$ & $\begin{array}{l}205 \\
430 \\
304 \\
287 \\
281\end{array}$ & $\begin{array}{l}201 \\
155\end{array}$ & $\begin{array}{l}196 \\
370 \\
359 \\
315 \\
298\end{array}$ & $\begin{array}{l}212 \\
530 \\
363 \\
254 \\
304\end{array}$ \\
\hline 73 & 59 & 76 & 74 & 211 & 141 & 235 & 211 & 295 & 191 & 312 & 317 \\
\hline $\begin{array}{l}70 \\
40 \\
55 \\
39 \\
29 \\
61 \\
\end{array}$ & $\begin{array}{l}42 \\
24 \\
68\end{array}$ & $\begin{array}{l}42 \\
39 \\
29 \\
38 \\
33 \\
39\end{array}$ & $\begin{array}{l}40 \\
41 \\
82 \\
30 \\
27 \\
43\end{array}$ & $\begin{array}{r}255 \\
181 \\
41 \\
51 \\
44 \\
115\end{array}$ & $\begin{array}{r}53 \\
56 \\
104\end{array}$ & $\begin{array}{r}168 \\
235 \\
50 \\
54 \\
47 \\
153\end{array}$ & $\begin{array}{r}77 \\
145 \\
33 \\
38 \\
25 \\
134\end{array}$ & $\begin{array}{l}468 \\
315 \\
220 \\
291 \\
502 \\
425\end{array}$ & $\begin{array}{l}213 \\
769 \\
472\end{array}$ & $\begin{array}{l}343 \\
215 \\
236 \\
377 \\
449 \\
322\end{array}$ & $\begin{array}{l}286 \\
382 \\
206 \\
311 \\
346 \\
234\end{array}$ \\
\hline 52 & 64 & 37 & 43 & 111 & 139 & 88 & 71 & 386 & 438 & 358 & 285 \\
\hline $\begin{array}{r}57 \\
156 \\
50 \\
44 \\
46\end{array}$ & $\begin{array}{l}71 \\
31 \\
27 \\
70\end{array}$ & $\begin{array}{r}13 \\
277 \\
34 \\
55 \\
34\end{array}$ & $\begin{array}{r}73 \\
128 \\
74 \\
53 \\
48\end{array}$ & $\begin{array}{r}226 \\
231 \\
187 \\
186 \\
71\end{array}$ & $\begin{array}{r}233 \\
187 \\
196 \\
35\end{array}$ & $\begin{array}{r}292 \\
538 \\
234 \\
182 \\
45\end{array}$ & $\begin{array}{l}158 \\
167 \\
150 \\
176 \\
116\end{array}$ & $\begin{array}{l}255 \\
369 \\
352 \\
149 \\
100\end{array}$ & $\begin{array}{l}367 \\
293 \\
176 \\
139\end{array}$ & $\begin{array}{r}118 \\
269 \\
225 \\
112 \\
81\end{array}$ & $\begin{array}{l}201 \\
390 \\
482 \\
183 \\
103\end{array}$ \\
\hline 49 & 49 & 38 & 63 & 163 & 180 & 166 & 145 & 206 & 252 & 127 & 256 \\
\hline
\end{tabular}

were found in the county boroughs, and the towns most affected were Dudley, Bristol, and Birmingham, where the rates were 1,050, 691, and 507 per million. 
TABLE

\begin{tabular}{|c|c|c|c|c|c|c|c|c|c|c|}
\hline & \multirow{2}{*}{\multicolumn{3}{|c|}{ Counties }} & & & & \multicolumn{4}{|c|}{1947} \\
\hline & & & & & & & County & C.B.s & U.D.s & R.D.s \\
\hline & $\begin{array}{l}\text { Cheshire } \\
\text { Lancashire }\end{array}$ & $\begin{array}{l}. . \\
. .\end{array}$ & $\because$. & $\begin{array}{l}\cdots \\
\cdots\end{array}$ & $\begin{array}{l}. \\
. .\end{array}$ &. & $\begin{array}{l}190 \\
214\end{array}$ & $\begin{array}{l}146 \\
213\end{array}$ & $\begin{array}{l}178 \\
215\end{array}$ & $\begin{array}{l}342 \\
221\end{array}$ \\
\hline \multirow[t]{2}{*}{ NORTH Western } & . & . & .. & .. & . & .. & 209 & 205 & 205 & 266 \\
\hline & $\begin{array}{l}\text { West Riding } \\
\text { East Riding } \\
\text { North Riding }\end{array}$ & $\because$ & $\begin{array}{l}. \\
\cdots \\
\cdots\end{array}$ & $\begin{array}{l}\cdots \\
\cdots \\
\therefore\end{array}$ & $\begin{array}{l}\because \\
\therefore \\
\therefore\end{array}$ & $\begin{array}{l}. \\
. .\end{array}$ & $\begin{array}{l}224 \\
290 \\
206\end{array}$ & $\begin{array}{l}187 \\
267 \\
136\end{array}$ & $\begin{array}{r}268 \\
325 \\
92\end{array}$ & $\begin{array}{l}284 \\
321 \\
421\end{array}$ \\
\hline \multirow[t]{2}{*}{ YORKSHIRE $\quad}$. & .. & .. & .. & .. & . & .. & 230 & 194 & 248 & 322 \\
\hline & $\begin{array}{l}\text { Durham } \\
\text { Northumberla } \\
\text { Cumberland } \\
\text { Westmorland }\end{array}$ & $\begin{array}{l}\text { and } \\
\ldots\end{array}$ & $\begin{array}{l}\ldots \\
\cdots \\
\cdots\end{array}$ & $\begin{array}{l}\ldots \\
\cdots \\
\cdots\end{array}$ & $\begin{array}{l}\ldots \\
\cdots \\
\cdots\end{array}$ & $\begin{array}{l}. . \\
\cdots \\
. .\end{array}$ & $\begin{array}{l}285 \\
231 \\
301 \\
367\end{array}$ & $\begin{array}{l}220 \\
202 \\
108\end{array}$ & $\begin{array}{l}333 \\
239 \\
242 \\
258\end{array}$ & $\begin{array}{l}310 \\
305 \\
443 \\
466\end{array}$ \\
\hline \multirow[t]{2}{*}{ NORTHERN } & .. & .. & .. & .. & .. & .. & 272 & 206 & 294 & 350 \\
\hline & $\begin{array}{l}\text { Brecknock } \\
\text { Carmarthen } \\
\text { Glamorgan } \\
\text { Monmouth }\end{array}$ & $\begin{array}{l}\cdots \\
\cdots \\
\cdots\end{array}$ & $\begin{array}{l}\ldots \\
\cdots \\
\cdots\end{array}$ & $\begin{array}{l}\ldots \\
\cdots \\
\cdots\end{array}$ & $\begin{array}{l}. \\
\cdots \\
\cdots\end{array}$ & $\begin{array}{l}. . \\
. . \\
. .\end{array}$ & $\begin{array}{r}282 \\
291 \\
147 \\
97\end{array}$ & $\begin{array}{l}129 \\
109\end{array}$ & $\begin{array}{r}254 \\
366 \\
134 \\
67\end{array}$ & $\begin{array}{l}294 \\
236 \\
227 \\
260\end{array}$ \\
\hline \multirow[t]{2}{*}{ WALES 1} & .. & .. & .. & .. & .. & .. & 153 & 125 & 134 & 240 \\
\hline & $\begin{array}{l}\text { Caernarvon } \\
\text { Cardigan } \\
\text { Denbigh } \\
\text { Flint .. } \\
\text { Merioneth } \\
\text { Montgomery } \\
\text { Pembroke } \\
\text { Radnor } \\
\text { Anglesey }\end{array}$ & $\begin{array}{l}. \\
\therefore \\
\therefore \\
. \\
\cdots \\
\therefore \\
\therefore \\
\therefore \\
\therefore\end{array}$ & $\begin{array}{l}\cdots \\
\therefore \\
\therefore \\
\therefore \\
\therefore \\
\therefore \\
\cdots \\
\therefore\end{array}$ & $\begin{array}{l}. \\
\therefore \\
\therefore \\
\therefore \\
\therefore \\
\therefore \\
\therefore \\
\therefore\end{array}$ & $\begin{array}{l}. \\
\therefore \\
\therefore \\
\therefore \\
\therefore \\
\therefore \\
\therefore \\
\therefore\end{array}$ & $\begin{array}{l}. . \\
\cdots \\
. .\end{array}$ & $\begin{array}{r}89 \\
228 \\
145 \\
274 \\
130 \\
335 \\
118 \\
356 \\
42\end{array}$ & & $\begin{array}{r}126 \\
338 \\
171 \\
376 \\
58 \\
116 \\
123 \\
320 \\
0\end{array}$ & $\begin{array}{r}39 \\
172 \\
123 \\
167 \\
188 \\
473 \\
114 \\
372 \\
68\end{array}$ \\
\hline WALES 2 & .. & .. & .. & . & . & .. & 173 & & 192 & 156 \\
\hline TOtal & . & .. & .. & .. & .. & .. & 221 & 199 & 211 & 239 \\
\hline
\end{tabular}

The distribution of poliomyelitis in some of these localized outbreaks has been quite different from that exhibited by other infectious diseases. Gilloran (1949), investigating the outbreak in Dorset in 1947, found that the better social classes had the highest attack rates, and that in the borough of Poole where the incidence was high the majority of the cases occurred in the better-class residential districts. A similar phenomenon was reported for Luton (Grundy, 1948), where poliomyelitis occurred "in circumstances of good health and low infection-a characteristic of poliomyelitis which seems to favour populations in favourable conditions where the public health services are above the average ". Outbreaks in other areas did not show this social distinction. Daley and Benjamin (1948) found an insignificant correlation between the proportion of males aged $14+$ who were in social classes IV and $\mathrm{V}$ and the attack rates of poliomyelitis in the 28 London boroughs. Hill and 
III-continued

\begin{tabular}{|c|c|c|c|c|c|c|c|c|c|c|c|}
\hline \multicolumn{4}{|c|}{1948} & \multicolumn{4}{|c|}{1949} & \multicolumn{4}{|c|}{1950} \\
\hline County & C.B.s & U.D.s & R.D.s & County & C.B.s & U.D.s & R.D.s & County & C.B.s & U.D.s & R.D.s \\
\hline $\begin{array}{l}43 \\
37\end{array}$ & $\begin{array}{l}47 \\
40\end{array}$ & $\begin{array}{l}34 \\
31\end{array}$ & $\begin{array}{l}70 \\
35\end{array}$ & $\begin{array}{l}114 \\
109\end{array}$ & $\begin{array}{l}56 \\
97\end{array}$ & $\begin{array}{l}128 \\
131\end{array}$ & $\begin{array}{l}204 \\
103\end{array}$ & $\begin{array}{l}120 \\
131\end{array}$ & $\begin{array}{l}132 \\
155\end{array}$ & $\begin{array}{r}123 \\
96\end{array}$ & $\begin{array}{l}83 \\
87\end{array}$ \\
\hline 38 & 41 & 32 & 48 & 110 & 92 & 131 & 141 & 129 & 152 & 103 & 85 \\
\hline $\begin{array}{l}40 \\
54 \\
28\end{array}$ & $\begin{array}{l}35 \\
65 \\
63\end{array}$ & $\begin{array}{l}42 \\
39 \\
15\end{array}$ & $\begin{array}{l}54 \\
39 \\
13\end{array}$ & $\begin{array}{r}220 \\
91 \\
63\end{array}$ & $\begin{array}{r}237 \\
88 \\
34\end{array}$ & $\begin{array}{r}209 \\
77 \\
39\end{array}$ & $\begin{array}{l}171 \\
113 \\
115\end{array}$ & $\begin{array}{l}171 \\
107 \\
207\end{array}$ & $\begin{array}{l}190 \\
105 \\
172\end{array}$ & $\begin{array}{l}123 \\
126 \\
187\end{array}$ & $\begin{array}{r}211 \\
94 \\
258\end{array}$ \\
\hline 40 & 41 & 38 & 42 & 188 & 206 & 176 & 148 & 168 & 179 & 132 & 205 \\
\hline $\begin{array}{l}32 \\
21 \\
40 \\
15\end{array}$ & $\begin{array}{l}23 \\
28 \\
46\end{array}$ & $\begin{array}{l}44 \\
15 \\
36 \\
32\end{array}$ & $\begin{array}{r}22 \\
20 \\
40 \\
0\end{array}$ & $\begin{array}{r}45 \\
124 \\
135 \\
286\end{array}$ & $\begin{array}{r}52 \\
177 \\
89\end{array}$ & $\begin{array}{r}37 \\
92 \\
59 \\
189\end{array}$ & $\begin{array}{r}49 \\
40 \\
209 \\
375\end{array}$ & $\begin{array}{r}179 \\
262 \\
135 \\
45\end{array}$ & $\begin{array}{l}171 \\
293 \\
149\end{array}$ & $\begin{array}{r}194 \\
194 \\
118 \\
31\end{array}$ & $\begin{array}{r}162 \\
378 \\
140 \\
58\end{array}$ \\
\hline 29 & 26 & 34 & 24 & 85 & 100 & 61 & 106 & 196 & 214 & 183 & 190 \\
\hline $\begin{array}{l}56 \\
94 \\
63 \\
26\end{array}$ & $\begin{array}{l}85 \\
29\end{array}$ & $\begin{array}{r}63 \\
153 \\
47 \\
22\end{array}$ & $\begin{array}{l}53 \\
51 \\
57 \\
46\end{array}$ & $\begin{array}{r}89 \\
76 \\
103 \\
85\end{array}$ & $\begin{array}{r}114 \\
9\end{array}$ & $\begin{array}{r}234 \\
85 \\
110 \\
105\end{array}$ & $\begin{array}{r}26 \\
70 \\
60 \\
138\end{array}$ & $\begin{array}{r}107 \\
216 \\
154 \\
78\end{array}$ & $\begin{array}{r}131 \\
85\end{array}$ & $\begin{array}{r}176 \\
310 \\
131 \\
76\end{array}$ & $\begin{array}{r}77 \\
150 \\
272 \\
69\end{array}$ \\
\hline 57 & 75 & 48 & 54 & 96 & 95 & 109 & 68 & 141 & 123 & 129 & 197 \\
\hline $\begin{array}{r}24 \\
19 \\
6 \\
51 \\
128 \\
66 \\
35 \\
49 \\
20\end{array}$ & & $\begin{array}{r}42 \\
0 \\
0 \\
43 \\
0 \\
56 \\
48 \\
0 \\
0\end{array}$ & $\begin{array}{r}0 \\
28 \\
11 \\
59 \\
229 \\
36 \\
22 \\
72 \\
33\end{array}$ & $\begin{array}{r}24 \\
37 \\
12 \\
83 \\
0 \\
66 \\
23 \\
99 \\
0\end{array}$ & & $\begin{array}{r}28 \\
108 \\
26 \\
99 \\
0 \\
0 \\
24 \\
154 \\
0\end{array}$ & $\begin{array}{r}19 \\
0 \\
0 \\
68 \\
0 \\
108 \\
22 \\
72 \\
0\end{array}$ & $\begin{array}{r}210 \\
56 \\
385 \\
194 \\
122 \\
502 \\
124 \\
444 \\
100\end{array}$ & & $\begin{array}{r}183 \\
0 \\
348 \\
141 \\
57 \\
669 \\
118 \\
463 \\
104\end{array}$ & $\begin{array}{r}247 \\
85 \\
416 \\
245 \\
171 \\
395 \\
130 \\
435 \\
98\end{array}$ \\
\hline 35 & & 27 & 42 & 35 & & 44 & 28 & 238 & & 214 & 258 \\
\hline 51 & 48 & 50 & 53 & 157 & 138 & 153 & 151 & 210 & 228 & 186 & 226 \\
\hline
\end{tabular}

Martin (1949) found no relation between the attack rate of poliomyelitis and the crude death rate from all causes, nor between the attack rate and the infant mortality rate in the 126 large towns and 148 smaller towns, nor between the attack rate of poliomyelitis and the overcrowding index in the London boroughs. Table III shows that, in general, the attack rate in a county is very similar in each density area. In 1950 the correlation coefficient between the attack rates in the urban and rural districts of the counties of England and Wales was 0.75.

\section{SOME EPIDEmiological Factors}

The spread of infection presents some interesting problems, since poliomyelitis, except in the more advanced stage of the disease, cannot be diagnosed with certainty and many minor attacks probably escape notice or do not require medical attention. 
Although in England and Wales the incidence of poliomyelitis has always begun to rise with the advent of warm weather and has reached epidemic proportions in the summer, its relationship to weather conditions is not very definite. The hot dry summer of 1949 might be held to favour the theory of a dust-borne infection, but the larger outbreak in the following year occurred in a wet cold summer.

An important contribution to knowledge of the incidence of the disease in the child population has been made by Casey and others (1950). In a field investigation carried out in Chicago, they showed that subclinical attacks in children were common. Poliomyelitis virus was recovered from the stools of 53 per cent. of contacts (i.e. children living in the immediate neighbourhood of a paralytic case), from 11 per cent. of non-contacts (i.e. children living in the surrounding neighbourhood of the first group), and from 8 per cent. of controls (i.e. children living ten blocks away from the patient with a clinical attack). The authors estimate that probably most children in Chicago had had one subclinical attack of poliomyelitis by their sixth birthday-either different strains or reinfections-and that among Chicago children paralysis was a condition that occurred less than once in 200 cases. We know that trauma can, at least sometimes, affect the course of the disease, for McCloskey (1950) found in Victoria, Australia, that a relation existed in a number of cases between an injection of an immunizing agent and the subsequent development of paralytic poliomyelitis. Hill and Knowelden (1950) found that the distribution of the bodily sites of paralysis was quite abnormal in children who had been inoculated within the month preceding the onset of their illness. In children recently inoculated, the injected limb (arm or leg) was much more frequently a site of paralysis than in children not recently inoculated. Hill and Knowelden concluded " that there may be present in the poliomyelitis group cases which would not have been clinically diagnosed as poliomyelitis at all if their inoculation had not brought them into the paralytic group ". The finding of Casey and his co-workers that poliomyelitis is generally a non-clinical condition from which clinical cases only rarely develop, is in agreement with many of the observed features of the trend of the disease. This would explain the rarity of multiple cases in a family, which has been generally noted in former epidemics, and the larger incidence in the better residential districts, which has been occasionally observed in some local outbreaks. It is well known that with infectious diseases of childhood the age of attack is correlated with density, i.e. children living in overcrowded conditions contract infectious diseases, on the average, at younger ages than their more fortunate brethren living in a better environment. This may well be the case with poliomyelitis; children living in the less congested district of an area may have been subjected to a much smaller risk of a subclinical attack than the remainder, and are consequently much more likely to develop a clinical attack during an outbreak.

The factors which determine whether paralysis develops or not have not been found. The evidence that inoculation can have this effect is fairly conclusive, but this factor can account for only an exceedingly small proportion of the total cases. Exercise in the pre-paralytic stage also appears to be of importance. It has been 
shown by several workers that the usual vital statistical indices of general living conditions are unrelated to the level of the incidence rate. Table III does not suggest that any particular area has a peculiar liability to a high incidence rate, nor that a high incidence in one outbreak is necessarily followed by a low incidence in a succeeding outbreak. To examine the latter point in more detail the attack rates in the 28 London boroughs in successive outbreaks were correlated, and the correlations were $0.273 \pm 0 \cdot 175$ for 1947 and 1949 , and $0.077 \pm 0 \cdot 188$ for 1949 and 1950.

Both correlation coefficients are insignificant, and it may therefore be stated that in these areas there was no relation between successive outbreaks.

It also seemed of interest to examine whether the variations in the incidence of acute poliomyelitis between areas were related to the prevalence of other infectious diseases. Accordingly, the notification rates of scarlet fever, whooping cough, diphtheria, and measles in the 28 London boroughs were correlated separately and in total with the notification rates of acute poliomyelitis. (See Table IV.)

TABLE IV

Correlations between Attack Rate from Poliomyelitis and other Infectious Diseases

\begin{tabular}{|c|c|c|c|c|c|c|}
\hline Year & .. & . & 1947 & 1948 & 1949 & 1950 \\
\hline \multirow{2}{*}{$\begin{array}{l}\text { Polio- } \\
\text { myelitis- } \\
\text { and }\end{array}$} & \multicolumn{2}{|l|}{$\begin{array}{l}\text { scarlet fever } \\
\text { whooping cough } \\
\text { diphtheria .. } \\
\text { measles .. }\end{array}$} & $\begin{array}{c}r \\
0.406 \pm 0.16 \\
0.175 \pm 0.18 \\
0.735 \pm 0.09 \\
-0.087 \pm 0.19\end{array}$ & $\begin{array}{c}r \\
0.051 \pm 0.18 \\
0.259 \pm 0.18 \\
0.428 \pm 0.15 \\
0.053 \pm 0.19\end{array}$ & $\begin{array}{r}r \\
-0.155 \pm 0.18 \\
0.093 \pm 0.18 \\
0.092 \pm 0.19 \\
-0.006 \pm 0.19\end{array}$ & $\begin{array}{c}r \\
0.522 \pm 0.14 \\
0.027 \pm 0 \cdot 19 \\
0 \cdot 040 \pm 0 \cdot 19 \\
0 \cdot 319 \pm 0 \cdot 17\end{array}$ \\
\hline & \multicolumn{2}{|c|}{$\begin{array}{l}\text { combined rate of } \\
\text { these four diseases }\end{array}$} & $0 \cdot 163 \pm 0 \cdot 18$ & $0.134 \pm 0.19$ & $-0.002 \pm 0.19$ & $0 \cdot 324 \pm 0 \cdot 17$ \\
\hline
\end{tabular}

The incidence of whooping cough and measles was unrelated to the incidence of poliomyelitis in each year, and the distribution of poliomyelitis followed the distribution of diphtheria in 1947 and 1948 and that of scarlet fever in 1947 and 1950. The combined incidence of the four infectious diseases showed no relation to poliomyelitis in any of the four years. Thus it appears that the incidence of poliomyelitis was unrelated to that of the common infectious diseases of childhood.

\section{SUMMARY}

The epidemic curve of poliomyelitis in England and Wales during 1947-50 showed considerable annual fluctuations.

In 1947 and 1950 the incidence rose steadily to a peak, but in 1949 the rise was not continuous and the maximum occurred several weeks later. In 1950 the rise in incidence began about a month earlier.

For England and Wales as a whole there is no evidence that the towns have a greater liability than the rural districts. The disease was more widely scattered in 1950 than in former years of high incidence, although in 1949 the northern counties and Wales had a relatively light experience. 
The area incidence of successive outbreaks did not appear to be related.

In the London boroughs the incidence was not related to that of infectious diseases of childhood.

\section{REFERENCES}

Bradley, W. H. (1950). Monthly Bull. M. of H. and P.H.L.S., 9, 203.

Casey, A. E., Fishbein, W. I., Schabel, F. M., and Smith, H. T. (1950). Amer. J. pub. Hlth, $40,1241$. Daley, A., and Benjamin, B. (1948). Med. Offr., 80, 171.

Gilloran, J. L. (1949). Ibid., 81, 255.

Grundy, F. (1948). Cited ibid., 79, 175.

Hill, A. Bradford, and Knowelden, J. (1950). Brit. med. J., $2,1$. and Martin, W. J. (1949). Ibid., 2, 357.

McCloskey, B. P. (1950). Lancet, 1, 659. 